\title{
Marketingcontrolling in der Unternehmenspraxis
}

\author{
Sven Reinecke und Alexander Tombach
}

\section{Zusammenfassung}

Der vorliegende Beitrag fasst die Ergebnisse einer umfassenden empirischen Studie zum Marketingcontrolling im deutschsprachigen Raum zusammen. Es wird analysiert, welche Instrumente, Verfahren und Kennzahlen des Marketingcontrollings in der Praxis tatsächlich eingesetzt werden - und ob sich besonders erfolgreiche Unternehmen von weniger erfolgreichen diesbezüglich unterscheiden. Es zeigt sich, dass in zahlreichen Bereichen des Marketingcontrollings nach wie vor große Umsetzungsherausforderungen bestehen. Mit anderen Worten: Bei der Implementierung von Marketingcontrolling herrscht eine Lücke zwischen verfügbaren theoretisch-konzeptionellen Ansätzen und der Anwendung in der unternehmerischen Praxis.

\section{Schlüsselwörter}

Marketingcontrolling $\cdot$ Return on Marketing · Marketing Performance

Measurement $\cdot$ Marketingkennzahlen $\cdot$ Marketingaccounting

\section{$1 \quad$ Einleitung}

Das Thema „Return on Marketing“ hat sich in den letzten zwanzig Jahren von einem Schnittstellen- bzw. Randbereich zu einem eigenständigen Themengebiet entwickelt, dessen Relevanz in Wissenschaft und Praxis unbestritten ist (u. a. Hanssens und Pauwels 2016). Wissenschaftler aus den Bereichen Marketing und Controlling sowie Unternehmensberater haben zahlreiche Verfahren und Ansätze entwickelt, die

\footnotetext{
S. Reinecke $(\bowtie) \cdot$ A. Tombach

Institut für Marketing und Customer Insight, Universität St. Gallen, St. Gallen, Schweiz

E-Mail: sven.reinecke@unisg.ch; alexander.tombach@unisg.ch
} 
Führungskräfte dabei unterstützen, Effektivität und Effizienz im Marketing sicherzustellen (Reinecke und Janz 2007; Bauer et al. 2016; Stewart und Gugel 2016). Der vorliegende Beitrag präsentiert die Ergebnisse einer empirischen Untersuchung im deutschsprachigen Raum. Dabei wurde untersucht, welche Instrumente, Verfahren und Kennzahlen des Marketingcontrollings in der Praxis tatsächlich eingesetzt werden. Die empirische Untersuchung wird seit Jahren regelmäßig von der Universität St. Gallen (HSG) durchgeführt, so dass es auch möglich ist, einige Veränderungen im Zeitverlauf aufzuzeigen. Ergänzend werden Unterschiede im Instrumenten- und Kennzahleneinsatz zwischen besonders erfolgreichen und weniger erfolgreichen Unternehmen ermittelt.

\section{Marketingcontrolling als Sicherstellen der Effektivität und Effizienz im Marketing}

Marketingcontrolling ist ein klassisches Schnittstellenthema zweier betriebswirtschaftlicher Teilgebiete. Marketing und Controlling stehen in einem ambivalenten Verhältnis zueinander. Einerseits werden sie als Zwillingsschwestern betrachtet, da beide übergreifende Konzepte sind, die nicht als Privileg einzelner Experten angesehen werden sollten (Deyhle 1988, S. 15). Anderseits besteht ein natürlicher Zielund Interessenskonflikt, wenn Marketing als Führung vom Markt her und Controlling als Führung vom Ergebnis her betrachtet wird.

Horváth (1985, S. 13) unterstreicht einen wesentlichen, allgemein akzeptierten Unterschied zwischen Marketing und Controlling: Marketing umfasst als unmittelbare Managementaufgabe die Entscheidungsfindung, während dem Controlling eine entscheidungsunterstützende Aufgabe zugeschrieben wird. Nachfolgend wird Marketing als Oberbegriff verwendet, der den Verkauf als Subinstrument einschließt insofern umfasst das Marketingcontrolling auch das Verkaufscontrolling. Marketingcontrolling sollte jedoch nicht mit Rechnungswesen im Marketing gleichgesetzt werden, auch wenn Letzteres eine wesentliche Informationsquelle ist. Die Funktion des Marketingcontrollings besteht vielmehr darin, die Effektivität (Wirksamkeit) und Effizienz (Wirtschaftlichkeit) einer marktorientierten Unternehmensführung sicherzustellen (Reinecke und Janz 2007, S. 38 f.).

Effektivität und Effizienz werden nachfolgend wie folgt verstanden: Effektivität bezeichnet im weiteren Sinn die Wirksamkeit und somit den Output der Leistungserstellung. Somit wird beschrieben, ob vorgegebene Ziele erreicht werden. Effektivität im engeren Sinn definiert den Wirksamkeitsgrad. Zentral ist die Frage, ob die Zielerreichung über einem vorab formulierten Zielniveau liegt. Effizienz hingegen bezeichnet den Grad der Wirtschaftlichkeit. Demnach ist eine Maßnahme effizient, wenn es keine andere Maßnahme gibt, die ein besseres Output/Input-Verhältnis erzielt (wobei das Verhältnis mindestens eins betragen muss) (siehe Abb. 1). Für eine ausführliche Begriffsdiskussion wird auf Lasslop (2003, S. 8 ff.) sowie Bonoma und Clark (1988) verwiesen. 


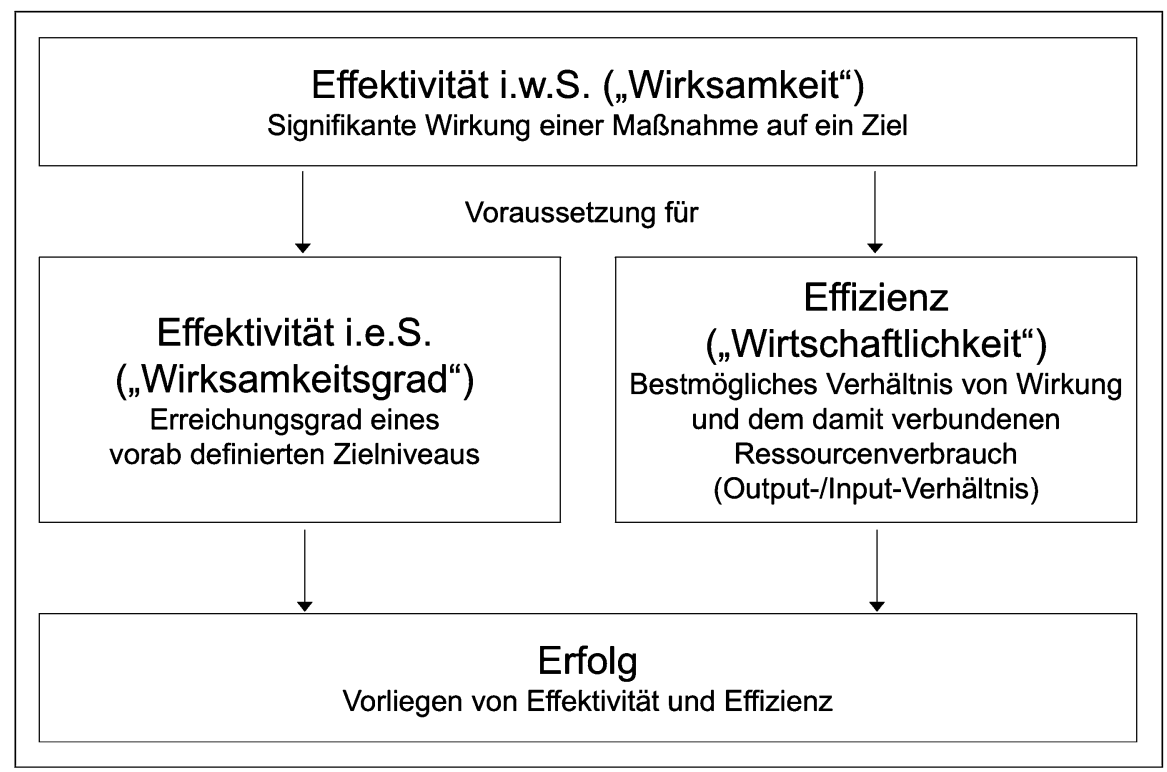

Abb. 1 Zusammenhang von Effektivität, Effizienz und Erfolg. (Quelle: Reinecke und Janz 2007, S. 39 in Anlehnung an Lasslop 2003, S. 12)

In der Marketingwissenschaft besteht unverändert großes Interesse am Thema Marketingcontrolling (u. v. a. Bauer et al. 2016; Katsikeas et al. 2016; Hanssens und Pauwels 2016; Bendle et al. 2020). Im Mittelpunkt der wissenschaftlichen Forschung steht zum einen die Beschreibung und der Nachweis der Wirkungskette von der Marketingaktivität bis zum unternehmerischen Erfolg, zum anderen die Definition und Auswahl geeigneter Marketingführungsgrößen.

Nachfolgend wird der aktuelle Stand des Marketingcontrollings in der betriebswirtschaftlichen Praxis kritisch evaluiert:

- Inwiefern verlangt die Unternehmensleitung einen Nachweis der Effektivität und Effizienz von Marketingmaßnahmen sowie der Gesamtfunktion „Marketing“? Welche Funktion wird dabei dem Marketingcontrolling zugeschrieben?

- Wie erfolgt die Planung von Marketing- und Verkaufsbudgets?

- Was sind die größten Herausforderungen des Marketingcontrollings?

- Welche Marketing- und Verkaufskennzahlen werden verwendet?

- Welche Marketingcontrollinginstrumente werden in welcher Form eingesetzt?

- Wodurch unterscheiden sich besonders erfolgreiche von weniger erfolgreichen Unternehmen hinsichtlich der Einsatzintensität von Marketingcontrollinginstrumenten?

- Inwiefern unterscheiden sich Groß- und Kleinunternehmen hinsichtlich der Einsatzintensität von Marketingcontrollinginstrumenten? 
Um diesen Fragen nachzugehen, hat das Institut für Marketing an der Universität St. Gallen im Sommer 2020 eine empirische Untersuchung durchgeführt. Nachfolgend wird der Studienaufbau kurz dargestellt, bevor die Ergebnisse präsentiert werden.

\section{$3 \quad$ Anlage der empirischen Studie}

Für die Studie wurden Führungskräfte im deutschsprachigen Raum mit dem Schwerpunkt Schweiz per E-Mail bzw. Web-Link kontaktiert und gebeten, an einer standardisierten schriftlichen Online-Befragung teilzunehmen. Die Grundgesamtheit der befragten Führungskräfte setzt sich aus Mitgliedern des Management Pools des Instituts für Marketing der Universität St. Gallen (Marketingführungskräfte aus dem deutschsprachigen Raum) sowie Mitgliedern des Swiss Marketing Panels zusammen. 179 Führungskräfte haben während des Erhebungszeitraums von Juni bis August 2020 an der Online-Befragung teilgenommen.

Die Mehrzahl der Befragten bekleidet eine leitende Funktion in ihrem Unternehmen. Geschäftsführer, Marketingleiter und Aufsichts- bzw. Verwaltungsräte machen insgesamt $65 \%$ der Befragten aus. Weitere $31 \%$ der Befragten sind Verkaufs- oder Werbeleiter. Die Befragten haben durchschnittlich eine Führungsverantwortung für 17 Mitarbeitende. Im Durchschnitt beschäftigen die befragten Unternehmen 1960 Mitarbeitende, wobei $39 \%$ Kleinunternehmen (<50 Mitarbeitende) und $28 \%$ Großunternehmen ( $>500$ Mitarbeitende) sind. Die Stichprobe weist ein breites Branchenspektrum auf. $44 \%$ der Unternehmen sind im Dienstleistungsbereich tätig, $22 \%$ im Industriegüterbereich, $19 \%$ in Handel \& Distribution sowie $15 \%$ im Konsumgüterbereich. Die Mehrzahl der Unternehmen aus der Stichprobe sind Kapitelgesellschaften (59 \% nicht börsennotierte, $22 \%$ börsennotiert). Die Studie erhebt keinen Anspruch auf Repräsentativität - weder für den deutschsprachigen noch für den schweizerischen Raum, auch wenn die Stichprobenstruktur der mittelständisch geprägten Wirtschaftslandschaft weitgehend entspricht (Tab. 1).

Tab. 1 Untersuchungsanlage. (Quelle: eigene Darstellung)

\begin{tabular}{l|l}
\hline Grundgesamtheit & $\begin{array}{l}\text { Mitglieder des Management Pools des Instituts für Marketing an der } \\
\text { Universität St. Gallen (HSG) und Mitglieder des Swiss Marketing Panels }\end{array}$ \\
\hline Stichprobe & $\begin{array}{l}\text { 179 Führungskräfte aus dem deutschsprachigen Raum, davon sind 142 im } \\
\text { Schweizer Markt, 68 im deutschsprachigen Raum, 23 in Europa und } \\
\text { 32 weltweit tätig (Mehrfachantworten möglich) }\end{array}$ \\
\hline Erhebungsverfahren & Schriftliche Onlinebefragung \\
\hline Erhebungszeitraum & Juni - August 2020 \\
\hline
\end{tabular}




\section{$4 \quad$ Stand des Marketingcontrollings in der Unternehmenspraxis}

\subsection{Marketingkultur}

Die Notwendigkeit, den Return on Marketing an das Top-Management zu berichten bzw. die Marketingeffizienz zu belegen, polarisiert stark. Bei einigen Unternehmen ist diese sehr hoch, bei anderen ist sie weniger ausgeprägt. Der Mittelwert der Nachweispflicht liegt bei 4,16 auf einer 7er-Skala ( 7 = höchste Nachweispflicht). Allerdings kann festgestellt werden, dass die Effizienz-Nachweispflicht in den letzten drei Jahren deutlich gestiegen ist $(\mathrm{MW}=4,79$ auf einer 7er Skala; $7=$ stark gestiegene Nachweispflicht) (vgl. Abb. 2). Die Beobachtung einer steigenden Nachweispflicht des Return on Marketing wird durch einen Vergleich mit zwei vergleichbaren Studien gestützt (Reinecke 2010, 2014), in denen die Nachweispflicht mit einem Mittelwert von 3,94 bzw. 4,14 niedriger eingeschätzt wurde.

Die Marketingleitung der meisten Unternehmen schreibt dem Marketing einen Investitionscharakter zu (MW = 5,7; Polaritätenskala: $1=$ Aufwand, $7=$ Investition). Dies überrascht nicht, weil Marketeers Marketing zunehmend (auch) als marktorientierte Unternehmensführung sehen (siehe Abb. 2). Der Mittelwert von 4,64 für die gleiche Einschätzung durch das Top-Management ist für das Marketingmanagement allerdings ein Silberstreif am Horizont. In einer früheren Studie von Reinecke (2010) hatte das Top-Management den Bereich Marketing noch deutlich stärker als Aufwand wahrgenommen $(\mathrm{MW}=4,18)$.

Für Marketingführungskräfte besteht weiterhin eine wichtige Handlungsaufforderung: Wenn dem Marketing Investitionscharakter zugeschrieben werden soll,

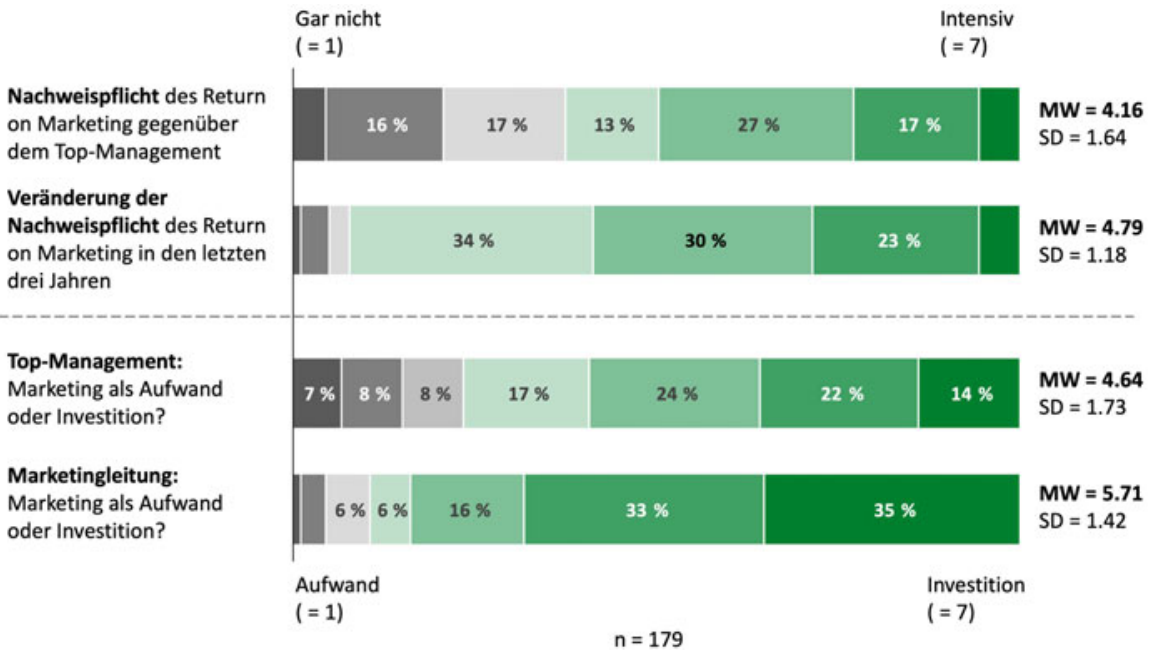

Abb. 2 Nachweispflicht des Return on Marketing und Einschätzung von Marketing als Aufwand oder Investition. (Quelle: eigene Darstellung) 
dann sollte dieser auch (finanzwirtschaftlich) nachgewiesen werden. Als Basis dafür ist es dazu erforderlich, geeignete Instrumente des Marketingcontrollings zur Erfassung von Effektivität und Effizienz der verschiedenen Marketingmaßnahmen des jeweiligen Unternehmens einzusetzen.

Die Erhebung zeigt jedoch, dass Marketingentscheidungen derzeit weniger datengestützt, sondern häufig intuitiv getroffen werden (siehe Abb. 3). Das bedeutet keineswegs automatisch, dass nicht-datengestützte Entscheidungen zu schlechteren Ergebnissen führen. Die Anwendung von Managementheuristiken kann in bestimmten Situationen sogar ein geeigneteres Entscheidungsverhalten bewirken (Gigerenzer und Brighton 2009). Jedoch ermöglicht es nur der gezielte Einsatz von Marketingcontrollinginstrumenten, die Wirksamkeit und die Wirtschaftlichkeit von Marketingmaßnahmen zu ermitteln, zu steuern und dem Top-Management gegenüber nachzuweisen. Die aus Sicht der Verfasser durchaus unzureichend datengesteuerte Marketingführung spiegelt sich in einer mäßigen internen Zufriedenheit mit dem Marketingcontrolling wider (Abb. 3). Der Mittelwert von 3,94 auf der Polaritätenskala (7 = höchste Zufriedenheit) impliziert, dass es Marketingführungskräften in der Praxis nur bedingt gelingt, Marketingeffektivität und -effizienz sicherzustellen. Rund $40 \%$ der Marketeers sind mit dem derzeitigen Stand des Marketingcontrollings unzufrieden.

Marketingführungskräfte haben die Relevanz des Marketingcontrollings erkannt und planen daher, zukünftig verstärkt Daten, Analysen und Fakten zur Unterstützung von Marketingentscheidungen einzusetzen (siehe Abb. 3). Die überlegte und gezielte Anwendung von Marketingcontrollinginstrumenten wird es Marketing-Führungskräften ermöglichen, der gestiegenen Effektivitäts- \& Effizienz-Nachweispflicht gerecht zu werden und das Marketing als wertstiftende Funktion im eigenen Unternehmen zu positionieren. Von einem echten wert- bzw. investitionsorientiertem Marketing ist die Marketingrealität jedoch noch weit entfernt.

Sehr unzufrieden

(1)
Sehr zufrieden

(7)

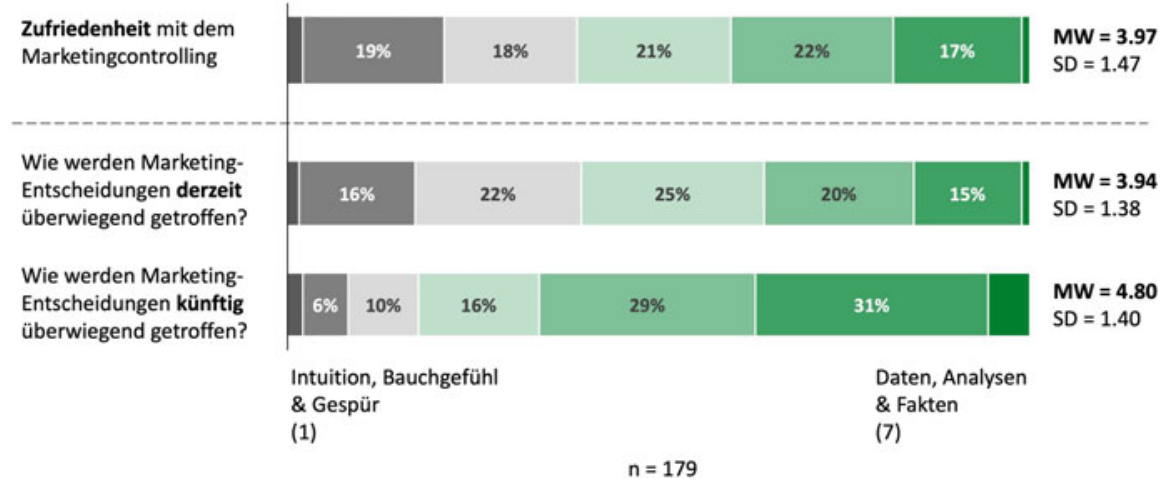

Abb. 3 Zufriedenheit mit dem Marketingcontrolling und Entscheidungsfindung im Marketing. (Quelle: eigene Darstellung) 


\subsection{Herausforderungen des Marketingcontrollings}

Im Rahmen der empirischen Erhebung wurden die wesentlichen Ursachen für die geringe Zufriedenheit mit dem Marketingcontrolling sowie die größten Herausforderungen erhoben. Die Ergebnisse zeigen, dass der konkrete Ursache-Wirkungsnachweis einzelner Marketingmaßnahmen und die Messbarkeit des Marketingerfolgs die größten Herausforderungen im Marketingcontrolling darstellen (siehe Abb. 4). Kenntnisse und Ressourcen in Datenanalyse und -auswertung sowie beim Datenzugang und -erhebung werden ebenfalls als große Herausforderungen betrachtet. Bemerkenswert ist hier, dass die Datenanalyse inzwischen sogar stärker als Engpass als die Datenerhebung wahrgenommen wird. Dies kann sicherlich auch auf die zunehmende Digitalisierung des Marketing und der damit verbundenen erhöhten Datenverfügbarkeit zurückzuführen zu sein. Des Weiteren ist es interessant, dass immer noch mehr als $50 \%$ der Unternehmen der Auffassung sind, dass die für das Marketingcontrolling zur Verfügung stehenden finanziellen Mittel unzureichend seien.

Hervorzuheben ist, dass es sich bei den genannten Herausforderungen fast ausschließlich um Bereiche handelt, die vom Marketingmanagement weitgehend selbst beeinflusst werden können. Der Vergleich mit Studienergebnissen aus dem Jahr 2014 (Reinecke 2014) zeigt, dass die Messbarkeit des Marketingerfolgs (MW = 5,61) sowie die Bereitstellung finanzieller Mittel (MW = 4,92) für das Marketingcontrolling heute eine geringere, wenn auch nach wie vor große Herausforderung für das Marketing darstellt.

Ein erster Schritt zu einer verbesserten Messbarkeit des Marketing wäre beispielsweise eine differenziertere Zielsetzung mittels eindeutig operationalisierter Kennzahlen. Marketingcontrolling im Sinne eines Regelkreiskonzepts ist ohne eine detaillierte Planung als Fundament gar nicht möglich, weil sich ohne Planungszielwerte keine

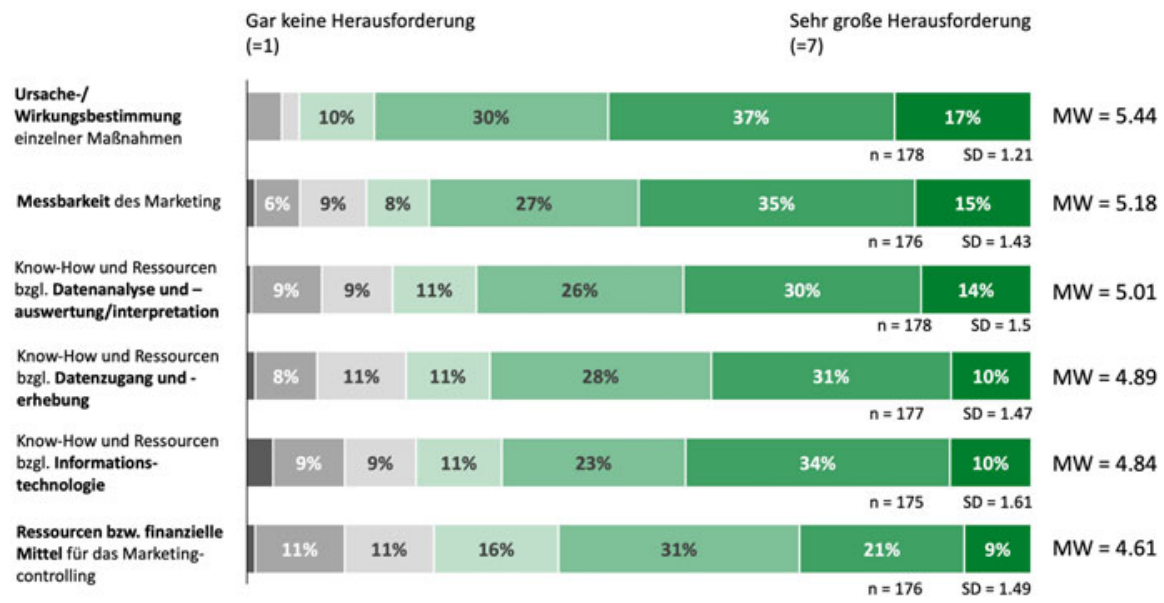

Abb. 4 Die größten Herausforderungen des Marketingcontrollings. (Quelle: eigene Darstellung) 
Abweichungen und auch keine Leistungsbeeinflussung feststellen lassen. Know-how und Ressourcen bezüglich Datenzugang einerseits sowie Datenanalyse \& -auswertung andererseits können durch gezielte Weiterbildungen der Mitarbeitenden oder den Erwerb von Know-how verbessert und internalisiert werden. Es ist anzunehmen, dass der Umgang mit Daten (künftig) als Kernkompetenz in einigen Bereichen des Marketing vorausgesetzt wird. Durch geeignete Investitionen in die Marktforschung und in Informationstechnologie kann zudem eine verbesserte und hochwertige Informations- und Datenbasis über Markt und Kunden geschaffen werden, die regelmäßige Analysen und Datenauswertungen erlaubt.

Somit unterstreichen die empirischen Ergebnisse erneut deutlich, dass nicht die Messbarkeit, sondern vielmehr die eindeutige Zuordnung von Ursache-Wirkungsbeziehungen die eigentliche Herausforderung des Marketing ist. Sofern im Marketing Umsatzziele dominieren und somit primär die Frage im Raum steht, welches Marketinginstrument (z. B. Werbung, Social Media-Kampagne, Messeauftritt, Preispromotion oder das Sponsoring) den Umsatzeffekt bewirkt hat, wird sich diese Herausforderung auch durch massive Investitionen in das instrumentelle Marketingcontrolling kaum lösen lassen. Letztlich sind hierfür Experimente erforderlich, die in der Praxis zumeist an den nicht kontrollierbaren, vielfältigen Feldeinflüssen sowie den damit verbundenen hohen Marktforschungskosten scheitern; im Bereich des digitalen Marketing sowie im Direct Marketing arbeitet man allerdings bereits hervorragend mit Experimenten zur Optimierung der operativen Maßnahmen (Stichwort: A/B-Tests, zum Teil sogar basierend auf künstlicher Intelligenz, siehe Reinecke 2020). Die fehlende Zurechenbarkeit des Leistungsbeitrags eines isolierten Marketinginstruments im realen Feld erhöht den Bedarf präzise Zwischenziele zu definieren (z. B. Besuchs- und Kontaktfrequenzen, Bekanntheit, Assoziationen, Einstellungen und Absichten), um die spezifischen Wirkungsbeiträge der einzelnen Instrumente zu belegen (siehe Reinecke und Geis 2020).

\subsection{Marketingbudgetierung}

Die Marketingbudgetierung ist eine wesentliche Aufgabe im Wechselspiel von Marketingplanung und -controlling, in deren Rahmen Unternehmen einerseits über die Budgethöhe insgesamt, andererseits über die Verteilung der für das Marketing einzusetzenden Ressourcen entscheiden.

Marketingmaßnahmen stehen aufgrund ihres hohen Anteils an den Gesamtkosten und ihres schwer zu erfassenden betriebswirtschaftlichen Nutzens in vielen Unternehmen verstärkt im Mittelpunkt von Rationalisierungsprozessen. Wie bereits dargelegt, fordert das Top-Management vermehrt den Ausweis und die Maximierung eines messbaren Beitrags des Marketing zum Unternehmenserfolg und -wert ein (Sheth und Sisodia 1995, S. 9 f., 2002, S. 349 f.). Wird das Marketingbudget dagegen einseitig als „Aufwand“ gesehen, dann besteht die Gefahr, dass beispielsweise eine geplante Herbstwerbekampagne einer Kostensenkungsmaßnahme im Frühjahr zum Opfer fällt. 
Die entscheidende Frage in diesem Zusammenhang ist zunächst jene nach der Budgethöhe: Welcher Betrag sollte für das Marketing investiert bzw. ausgegeben werden? Dabei ist es für das Top-Management von hoher Relevanz, inwiefern das eigene Unternehmen zu viel oder zu wenig Budgetmittel bereitstellt. Daher wurde im Rahmen der Studie überprüft, ob die Marketing- und Verkaufsführungskräfte hinsichtlich ihres derzeitigen Marketingbudgets Effizienzlücken erkennen. In zwei Fragen wurde das von den Führungskräften geschätzte Einsparpotenzial des Marketingbudgets (ohne wesentliche Wirkungsverluste) sowie das Optimierungspotenzial im Marketing bei gleichem Mitteleinsatz ermittelt. Das subjektive Einsparpotenzial im Marketing (siehe Abb. 5) wird mit durchschnittlich 13,1\% etwas niedriger eingeschätzt als das Optimierungspotenzial mit durchschnittlich 15,2\% bei gleichem Mitteleinsatz (Abb. 6). Zwar fällt diese Effizienzlücke erheblich geringer aus als in der dem berühmten US-amerikanischen Einzelhändler John Wanamaker zugeschriebenen Aussage «Die Hälfte des Geldes, die ich für Werbung ausgebe, ist zum Fenster herausgeworfen - leider weiß ich nicht, welche Hälfte es ist.» Dennoch offenbaren die empirischen Ergebnisse erhebliche Effizienzreserven, die sich mittels verbessertem Marketingcontrolling erschließen ließen - zumal sich auch gegenüber 2014 mit einem durchschnittlichen Einsparpotenzial von 14,7 \% (Reinecke 2014) keine wesentlichen Verbesserungen zeigen.

Da Unternehmen in der Regel mehrere Ansätze und Methoden zur Planung der Höhe des (aggregierten) Marketing- \& Verkaufsbudgets heranziehen und kombinieren (Reinecke und Janz 2007, S. 127 ff.; Fuchs 2008), wurden die Befragten gebeten,

Frage: Wie viel Prozent des Marketingbudgets könnten aus Ihrer Sicht eingespart werden, ohne die Marketingleistung Ihres Geschäftsbereichs maßgeblich zu beeinträchtigen?

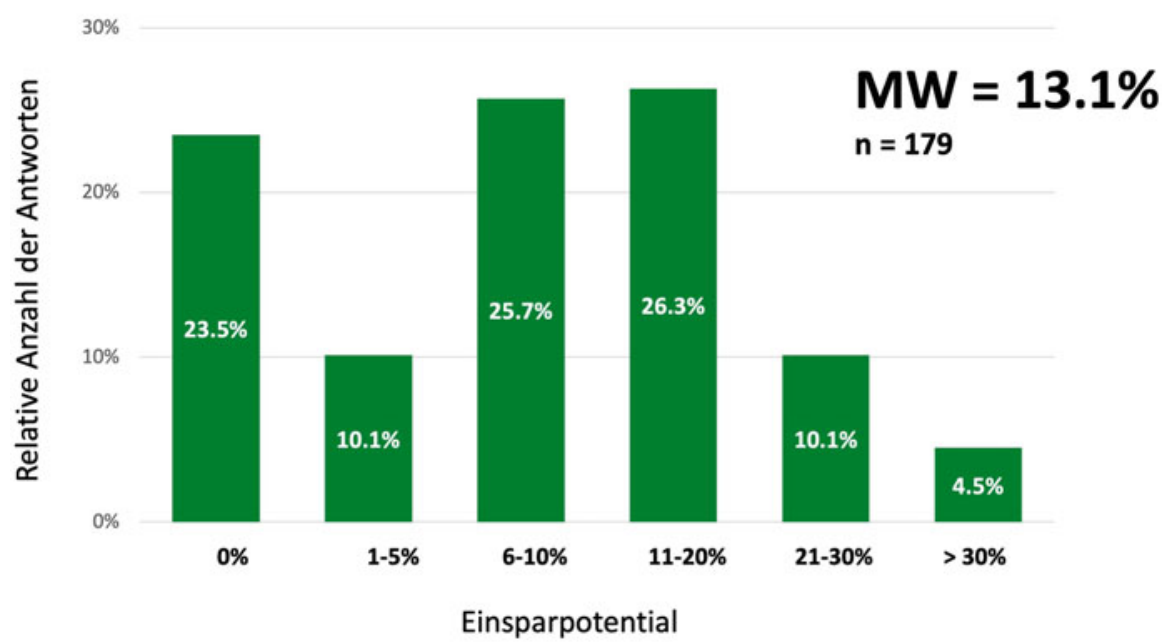

Abb. 5 Geschätztes Einsparpotenzial bei der Marketingbudgetfestlegung. (Quelle: eigene Darstellung) 
Frage: Um wie viel Prozent ließe sich das betriebswirtschaftliche Ergebnis Ihres Geschäftsbereichs Ihrer Meinung nach durch eine Optimierung des Mitteleinsatzes im Marketing noch steigern (bei gleicher Budgethöhe)?

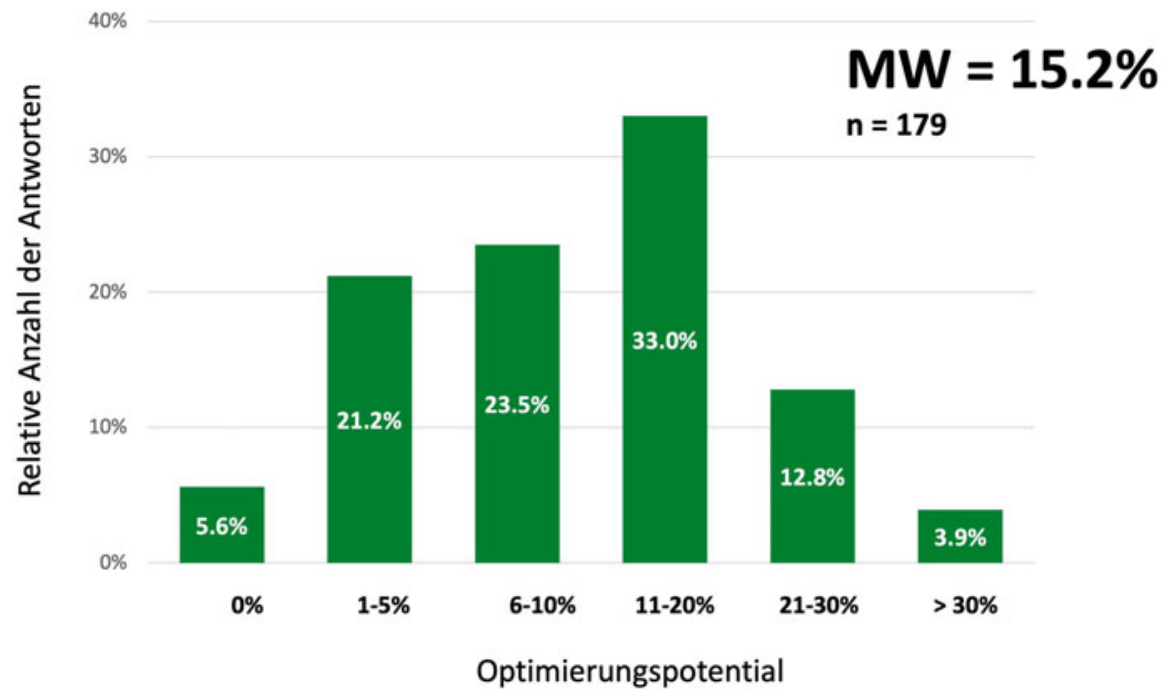

Abb. 6 Geschätztes Optimierungspotenzial im Marketing bei gleichem Mitteleinsatz. (Quelle: eigene Darstellung)

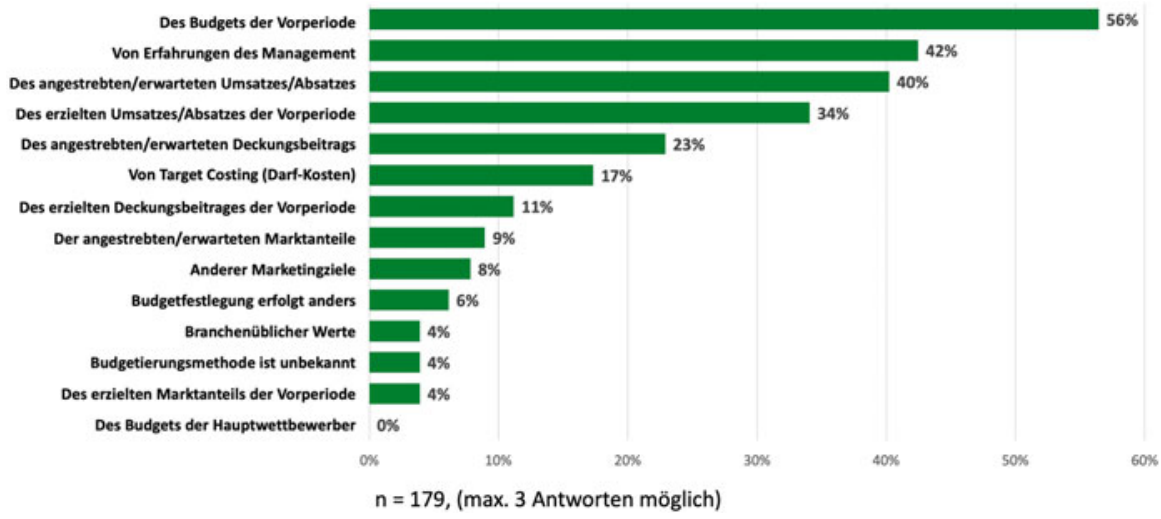

Abb. 7 Einsatz von Budgetmethoden und -ansätzen in der Unternehmenspraxis. (Quelle: eigene Darstellung)

bis zu drei der von ihnen primär genutzten Ansätze und Methoden zu nennen. Das Ergebnis (siehe Abb. 7) zeigt, dass die Marketingbudgetierung in der Unternehmenspraxis stark durch inkrementelle, induktive und heuristische Verfahren geprägt ist. Die Marketingbudgetfestlegung orientiert sich bei $56 \%$ der Unternehmen am Budget der Vorperiode. Bei dieser Methode handelt es sich um einen vergangen- 
heitsorientierten und wenig ergebnisorientierten Fortschreibungsansatz. Zudem beruht die Budgetierung in $42 \%$ der Unternehmen auf den Erfahrungen des Managements, deren Rationalität schwer zu überprüfen ist. Es ist daher zu vermuten, dass Bauchgefühl und politische Machtkalkulation die Höhe des Marketingbudgets stark beeinflussen. Prozentmethoden (beispielsweise Orientierung am Vorjahresumsatz bzw. -absatz, $34 \%$ ) werden ebenfalls bei zahlreichen befragten Unternehmen angewandt. Bei dieser Methode besteht jedoch die Gefahr einer prozyklischen Marketingbudgetierung, weil das Budget einseitig von einer Bezugsgröße bestimmt wird: Bei hohem Umsatz steht auch ein hohes Marketingbudget zur Verfügung, bei geringem Umsatz wird es reduziert. Differenzierte, zielorientierte Methoden, die über Umsatz oder Absatz hinausgehen, werden hingegen seltener angewendet. Wettbewerbsbezogene Budgetierungsansätze, das heißt eine Ausrichtung des Budgets an einem branchenüblichen Wert, werden dagegen kaum (4\%) oder gar nicht (Ausrichtung am Budget des Hauptwettbewerbers) genutzt. Letzteres dürfte darauf zurückzuführen sein, dass diese Werte außerhalb der marktforscherisch besonders gut erschlossenen Konsumgüterbranchen lediglich mit großem (finanziellen) Aufwand zu ermitteln und schwer zu vergleichen sind.

Die Ergebnisse entsprechen weitgehend der vergleichbaren Erhebung aus dem Jahr 2014 (Reinecke 2014) und verdeutlichen, dass Ansatz- und Methodenwahl in der Unternehmenspraxis weitgehend unverändert geblieben sind. Die empirischen Ergebnisse unterstreichen somit, dass eine begründete, rationalitätsgesteuerte Marketingbudgetierung eine der größten Defizitbereiche von Marketingplanung und -controlling bleibt.

\subsection{Zielpriorisierung im Marketing}

Die differenzierte Zielsetzung im Marketing mittels eindeutig operationalisierter Kennzahlen ist eine wesentliche Aufgabe des Marketingcontrollings. Im Rahmen der Studie wurde mittels Konstantsummenverfahren ermittelt, wie verschiedene Ziele im Marketing gewichtet werden. Da in der Unternehmenspraxis unterschiedliche Zielsysteme Anwendung finden, wurden die befragten Unternehmen gebeten, die Wichtigkeit ihrer Ziele anhand von drei Verfahren zu charakterisieren.

Zum einen wurde anhand der klassischen Balanced Scorecard von Kaplan und Norton (1992) ermittelt, wie finanzwirtschaftliche Ziele, markt- \& kundenorientierte Ziele, mitarbeiter- \& prozessorientierte Ziele sowie innovationsorientierte Ziele in den Unternehmen priorisiert werden (siehe Abb. 8). Dazu wurden die Befragten gebeten, 100 Punkte so auf die vier Dimensionen zu verteilen, dass sie die jeweilige Zielbedeutung widerspiegeln. Die Ergebnisse unterstreichen die hohe Bedeutung finanzwirtschaftlicher Ziele. Diese werden mit durchschnittlich 37 \% stärker als die markt- und kundenorientierten Ziele (29 \%) gewichtet. Bei den börsennotierten Unternehmen der Stichprobe haben die finanzwirtschaftlichen Ziele mit durchschnittlich $43 \%$ eine noch stärkere Bedeutung. Die mitarbeiter- \& prozessorientierten Ziele (18\%) werden ebenso wie die Innovationsziele (16\%) deutlich niedriger gewichtet. Die Zielgewichtung unterscheidet sich deutlich von vergleichbaren älte- 


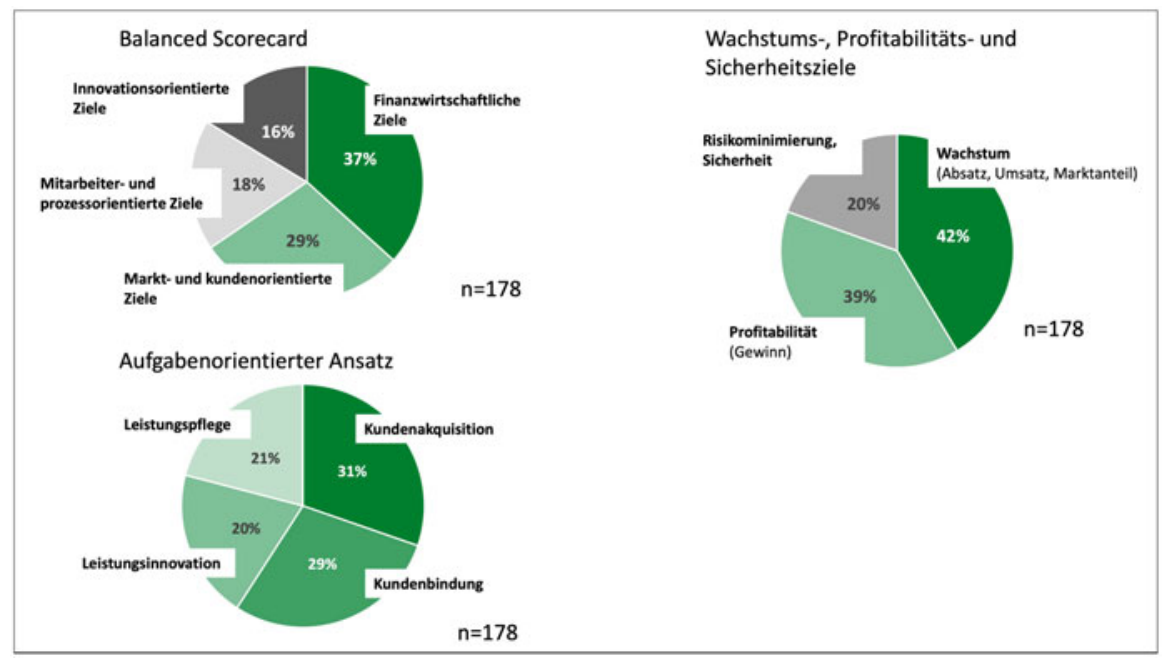

Abb. 8 Einschätzung der Bedeutung unternehmerischer Ziele. (Quelle: eigene Darstellung)

ren Untersuchungen (siehe Reinecke 2004, S. 146), bei denen die markt- und kundenorientierten Ziele noch die erste Stelle einnahmen. Dieses Ergebnis offenbart, dass selbst bei Marketingführungskräften die Finanzziele eindeutig dominieren. Zusätzlich ist anzunehmen, dass diese Einschätzung bei einer Befragung von CEOs und Finanzchefs noch deutlich ausgeprägter wäre. Von einer ,ausgewogenen“ (=balanced) Scorecard kann in der Realität somit nicht ausgegangen werden. Vielmehr hat sich die Bedeutung der Finanzziele sogar erhöht.

Des Weiteren wurde die relative Bedeutung von Wachstums-, Profitabilitäts- und Risikominimierungszielen ermittelt (siehe Abb. 8). Die Befragten verfügten erneut über 100 Punkte und wurden gebeten, diese so auf die drei Kategorien zu verteilen, dass sie die relative Bedeutung der drei Zielbereiche in ihrem Geschäftsbereich bestmöglich charakterisieren. Wachstumsziele (42\%) werden in den befragten Unternehmen priorisiert, jedoch nur unwesentlich höher als Profitabilitätsziele (39 \%). Risikominimierungsziele (20\%) werden weniger stark gewichtet. Die starke Ausrichtung auf Wachstumsziele ist bei einer Befragung von stark auf den Wettbewerb ausgerichteten Marketingführungskräften nicht verwunderlich. Die fast gleichwertige Gewichtung von Profitabilitätszielen impliziert allerdings, dass die gestiegene Effizienz-Nachweispflicht in den befragten Unternehmen zu einer Art Emanzipation der Profitabilitätsziele geführt hat. Eine noch stärkere Gewichtung von Profitabilitätszielen in einem traditionell von Umsatzzielen dominierten Marketing erleichtert es, Marketing weiter zu einer wertstiftenden betriebswirtschaftlichen Investition zu entwickeln.

Abschließend wurde anhand des aufgabenorientierten Ansatzes (Tomczak und Reinecke 1996; Tomczak et al. 2014) die durchschnittliche Bedeutung der vier 
Marketing-Kernaufgaben Kundenakquisition, Kundenbindung, Leistungspflege und Leistungsinnovation ermittelt. Dafür wurden die Befragten wiederum gebeten, 100 Punkte auf die vier Kernaufgaben gemäß ihrer derzeitigen unternehmensspezifischen Gewichtung zu verteilen. Die Kundenakquisition (31\%) und die Kundenbindung (29 \%) werden stärker gewichtet als die Leistungspflege (21\%) und die Leistungsinnovation (20\%). Der etwas stärkere Fokus auf das Erschließen und Ausschöpfen von Kundenpotenzialen ist vermutlich auf den hohen Anteil an Vertriebsgesellschaften in der Stichprobe zurückzuführen.

\subsection{Marketing- und Verkaufskennzahlen}

Marketingkennzahlen werden von Unternehmen zur Steuerung von Marketing und Verkauf eingesetzt. Um zu überprüfen, welche Kennzahlen prioritär eingesetzt werden, wurden die Unternehmen zu ihrem Kennzahleneinsatz befragt. Zudem wurde ermittelt, welche fünf der jeweils erhobenen Kennzahlen aus Führungskräftesicht die höchste Relevanz für den eigenen Geschäftsbereich haben.

Ähnlich wie in Vorgängeruntersuchungen (Reinecke 2004; Reinecke und Eberharter 2010; Reinecke 2014) zeigt sich, dass hauptsächlich absatz- oder finanzwirtschaftliche Marketing- und Verkaufskennzahlen wie beispielsweise Umsatz, Nettogewinn und Umsatzrentabilität verwendet werden (siehe Abb. 9). Kundenorientierte Ergebniskennzahlen wie Kundenzufriedenheit und Inputkenngrößen wie Kundenfrequenz/

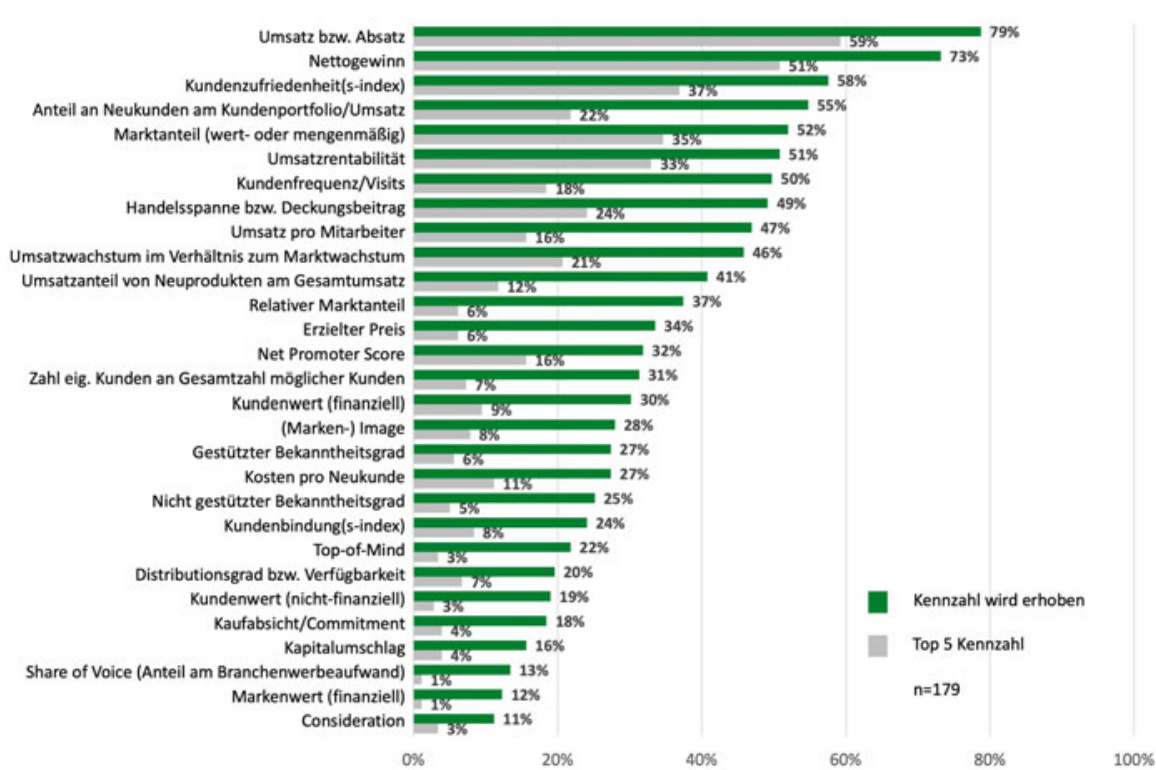

Abb. 9 Kennzahleneinsatz in Marketing und Verkauf. (Quelle: eigene Darstellung) 
Visits werden ebenfalls erhoben, allerdings seltener als Top-Steuerungskennzahl eingesetzt.

Konkurrenzorientierte Kenngrössen werden (mit Ausnahme des Marktanteils) kaum erhoben. Insbesondere wird selten auf komplexere, differenziertere Marketingkennzahlen wie Kunden- und Markenwert, Consideration oder Commitment zurückgegriffen. Vielmehr sind die Schlüsselkennzahlen stark finanzwirtschaftlich und „konservativ“ geprägt. Somit unterstreichen die empirischen Ergebnisse erneut eine Grundherausforderung des Marketingcontrollings: Kennzahlen sollten einerseits möglichst leicht verfügbar und daher hinsichtlich ihrer Definition bzw. Operationalisierung nicht $\mathrm{zu}$ kompliziert sein. Andererseits sind Marketingkennzahlen nur bedingt geeignet, wenn sie einseitig und beispielsweise ausschließlich vergangenheitsorientiert sind. So reicht es nicht aus, Umsatz als einzige Marketing- und Verkaufskennzahl zu wählen: Umsatz misst nur das Unternehmensziel „Wachstum“ (nicht aber Profitabilität und Sicherheit), und ist außerdem eine vergangenheitsorientierte Größe. Wenn der Umsatz nicht erreicht wird, dann ist es bereits meist zu spät, um proaktiv Maßnahmen zu ergreifen. Kennzahlen gewinnen auch immer nur durch Vergleiche Aussagekraft (Siegwart et al. 2010, S. 13 ff.): Entweder durch Zeitreihenanalysen oder durch Konkurrenzvergleiche. Isolierte absolute Kenngrößen ohne Vergleichsmöglichkeiten sind dagegen wenig zielführend und nicht handlungsorientiert.

Mittelwertvergleiche der Zufriedenheit mit dem Marketingcontrolling in Bezug auf die Verwendung von Marketingkennzahlen zeigen, dass der Einsatz von kundenorientierten Kennzahlen signifikant mit einer höheren Zufriedenheit mit dem Marketingcontrolling einhergeht (Abb. 10). Insbesondere sind Unternehmen, die den Kundenbindungsindex, den finanziellen Kundenwert und den Kundenanteil an der Anzahl möglicher Kunden messen, signifikant zufriedener mit dem unternehmensinternen Marketingcontrolling als diejenigen Unternehmen, die auf die Erhebung dieser Kennzahlen verzichten.

\begin{tabular}{|c|c|c|c|c|c|c|}
\hline \multirow[b]{2}{*}{ Kennzahl } & \multicolumn{2}{|c|}{$\begin{array}{l}\text { Mittelwert } \\
\text { Zufriedenheit mit dem } \\
\text { Marketingcontrolling }\end{array}$} & \multirow[b]{2}{*}{ Differenz } & \multirow[b]{2}{*}{ T-Test } & \multirow[b]{2}{*}{ Sig. } & \multirow[b]{2}{*}{$\begin{array}{c}\text { Signifikanzniveau } \\
\text { signifikant }(p<=0.05) \\
* \text { hochsignifikant }(p<=0.01) \\
* * \text { höchstsignifkant }(p<=0.001)\end{array}$} \\
\hline & $\begin{array}{c}\text { Kennzahl } \\
\text { verwendet }\end{array}$ & $\begin{array}{c}\text { Kennzahl } \\
\text { nicht } \\
\text { verwendet }\end{array}$ & & & & \\
\hline Kundenanteil an Anzahl möglicher Kunden & 4.55 & 3.70 & 0.849 & 3.690 & 0.000 & $* * *$ \\
\hline Kundenzufriedenheit & 4.23 & 3.62 & 0.615 & 2.817 & 0.005 & $* *$ \\
\hline Net Promoter Score & 4.46 & 3.75 & 0.710 & 3.082 & 0.002 & 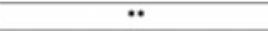 \\
\hline Kundenbindungsindex & 4.70 & 3.74 & 0.955 & 3.854 & 0.000 & $* * *$ \\
\hline Kundenwert Finanziell & 4.59 & 3.70 & 0.889 & 3.853 & 0.000 & $* * *$ \\
\hline Top of Mind & 4.56 & 3.81 & 0.757 & 2.902 & 0.004 & $*$ \\
\hline Ungestützter Bekanntheitsgrad & 4.47 & 3.81 & 0.661 & 2.652 & 0.009 & $*$ \\
\hline Gestützter Bekanntheitsgrad & 4.45 & 3.79 & 0.657 & 2.711 & 0.007 & $*$ \\
\hline Kaufabsicht / Commitment & 4.59 & 3.84 & 0.757 & 2.685 & 0.008 & $*$ \\
\hline Kundenfrequenz / Visits & 4.28 & 3.67 & 0.614 & 2.850 & 0.005 & $*$ \\
\hline Markenwert Finanziell & 4.73 & 3.87 & 0.861 & 2.614 & 0.010 & $* *$ \\
\hline Kosten pro Neukunde & 4.47 & 3.78 & 0.685 & 2.832 & 0.005 & 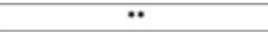 \\
\hline
\end{tabular}

Abb. 10 Zufriedenheit mit dem Marketingcontrolling in Bezug auf die Verwendung von Kennzahlen. (Quelle: eigene Darstellung) 
Im Rahmen der Erhebung wurde zudem untersucht, inwiefern der Einsatz spezifischer Kennzahlen mit dem (subjektiv eingeschätzten) betriebswirtschaftlichen Erfolg einhergeht. Dazu wurden die Führungskräfte zunächst gebeten anzugeben, wie sie ihre Zielerreichung bezüglich der Dimensionen der Balanced Scorecard, des aufgabenorientierten Ansatzes sowie von Wachstums-, Profitabilitäts- und Sicherheitszielen in den letzten drei Jahren einschätzen. Anschließend wurde ein Mittelwertvergleich der jeweiligen (subjektiven) Zielerreichung in Bezug auf die verwendeten Kennzahlen durchgeführt. Somit konnte ermittelt werden, inwiefern Unternehmen, die bestimmte Kennzahlen einsetzen, ihre Zielerreichung hinsichtlich der einzelnen Dimensionen höher einschätzen als solche, die auf den Einsatz der Kennzahlen verzichten.

Die Ergebnisse aus Abb. 11 lassen einen positiven Wirkungszusammenhang zwischen dem Einsatz bestimmter Kennzahlen und der Erreichung betriebswirtschaftlicher Ziele vermuten. Allerdings sei ausdrücklich darauf hingewiesen, dass hier kein einfacher Ursache-Wirkungszusammenhang angenommen werden kann. Kennzahleneinsatz und betriebswirtschaftlicher Erfolg gehen Hand-in-Hand miteinander, aber der Kennzahleneinsatz allein ist sicherlich nicht ursächlich für den Erfolg. Ein solcher Ursache-Wirkungszusammenhang ließe sich lediglich experimentell im Zeitablauf im Rahmen eines Panels nachweisen, nicht aber mit der Analyse zeitpunktbezogener Befragungsdaten wie in der vorliegenden Studie. Auch ein Vergleich mit den älteren Studien ist nicht sinnvoll, weil es sich um letztlich um eine Mehrwellenstudie und somit andere Unternehmen in der jeweiligen Stichprobe handelt.

Die Analyse des Zusammenhangs von Kennzahleneinsatz und dem subjektiveingeschätzten Erfolg bezüglich der drei Zielsysteme zeigt, dass sowohl wachstumsorientierte als auch effizienzorientierte, relative Kenngrößen mit den jeweiligen

\begin{tabular}{|c|c|c|}
\hline Kennzahl & Zieldimension & $\begin{array}{c}\text { Signifikanzniveau } \\
\text { *signifikant }(p<=0.05) \\
* * \text { hochsignifikant }(p<=0.01) \\
* * * \text { höchstsignifkant }(p<=0.001)\end{array}$ \\
\hline \multirow{3}{*}{ Kosten pro Neukunde } & Prozessorientierte Ziele & $* *$ \\
\hline & Innovationsorientierte Ziele & $* * *$ \\
\hline & Risikominimierung & $* * *$ \\
\hline \multirow{2}{*}{ Umsatzwachstum im Vergleich zum Marktwachstum } & Kundenbindung & $* *$ \\
\hline & Wachstum & $*$ \\
\hline \multirow{2}{*}{ Marktanteil } & Kundenorientierte Ziele & $*$ \\
\hline & Wachstum & $* *$ \\
\hline \multirow{2}{*}{ Neukundenanteil am Kundenportfolio/Umsatz } & Finanzwirtschaftliche Ziele & $*$ \\
\hline & Profitabilität & $*$ \\
\hline \multirow{2}{*}{ Kundenanteil an Gesamtzahl möglicher Kunden } & Leistungsinnovation & $\cdots$ \\
\hline & Leistungspflege & $*$ \\
\hline Umsatzrentabilität & Profitabilităt & $*$ \\
\hline Erzielter Preis & Kundenbindung & $* * *$ \\
\hline Share of Voice & Leistungsinnovation & $* *$ \\
\hline Markenimage & Leistungsinnovation & $* *$ \\
\hline Markenwert Finanziell & Leistungsinnovation & $* * *$ \\
\hline
\end{tabular}

Abb. 11 Kennzahleneinsatz und subjektive Zielerreichung anhand der Dimensionen der Balanced Scorecard, des aufgabenorientierten Ansatzes und von Wachstums-, Profitabilitäts- und Sicherheitszielen. (Quelle: eigene Darstellung) 
Erfolgsdimensionen in einem positiven Zusammenhang stehen. Insbesondere sticht die Kennzahl Kosten pro Neukunde hervor, deren Einsatz mit dem Erreichen dreier Zieldimensionen in einem positiven Zusammenhang steht. Zudem fällt auf, dass Unternehmen, die komplexere Kennzahlen wie Share of Voice, Markenimage und den finanziellen Markenwert ermitteln, erfolgreicher hinsichtlich der Erreichung ihrer Leistungsinnovationsziele sind.

Es muss hier ausdrücklich darauf hingewiesen werden, dass viele der aufgeführten nicht-finanziellen Kenngrößen lediglich von wenigen Unternehmen eingesetzt werden, so dass sich aufgrund der sich daraus ergebenen niedrigen Anzahl an Nennungen kein statistisch signifikanter Unterschied bezüglich des Kennzahleneinsatzes nachweisen lässt. Da diese häufig als absolute Effektivitätsziele verwendet werden, lässt sich aus den Daten somit nicht schließen, dass sie für den betriebswirtschaftlichen Erfolg weniger wichtig als Effizienzkennzahlen sind.

\subsection{Instrumente des Marketingcontrollings}

Kennzahlen und Kennzahlensysteme sind lediglich ein Baustein eines umfassenden Marketingcontrolling-Systems; sie ergänzen, aber ersetzen keineswegs strategische und operative Controllinginstrumente. Gerade im Marketing gibt es zahlreiche Bereiche, die mit Kennzahlen allein nur unzureichend abgedeckt werden können (Reinecke 2010, S. 81). Deshalb stellt sich die Frage, mit welchen Instrumenten und Verfahren Marketingführungskräfte versuchen, den Erfolg des Marketing zu steuern und zu belegen. Nachfolgend wird die Nutzung strategischer, operativer und finanzieller Instrumente des Marketingcontrollings in der Unternehmenspraxis dargestellt.

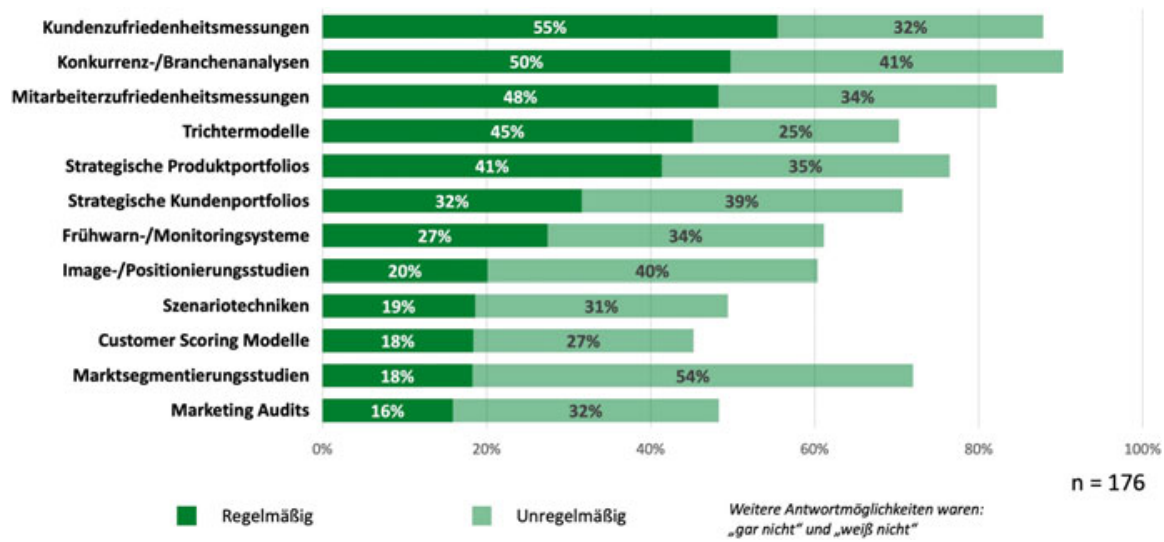

Abb. 12 Einsatzintensität strategischer Marketingcontrollinginstrumente. (Quelle: eigene Darstellung) 


\subsubsection{Strategisches Marketingcontrolling}

In der Unternehmenspraxis werden traditionelle und bewährte Instrumente wie Konkurrenz-, Branchen- und Kundenzufriedenheitsanalysen am häufigsten eingesetzt (siehe Abb. 12). Strategische Produkt- und Kundenportfolios finden ebenfalls regelmäßig Anwendung und werden durchschnittlich bei bereits sieben von zehn Unternehmen zumindest unregelmäßig eingesetzt. Ein Vergleich mit einer früheren Untersuchung (Reinecke 2014) zeigt jedoch, dass strategische Produktportfolios wieder häufiger als strategische Kundenportfolios zum Einsatz kommen. Dies verwundert angesichts der proklamierten verstärkten „Customer Centricity“ vieler Unternehmen sowie ihrer vermehrten Ausrichtung auf Customer Relationship Management (CRM) bzw. (Key) Account Management (KAM). Produkte sind somit nach wie vor die dominierende Dimension im Marketingcontrolling.

Ferner werden Trichtermodelle in über $70 \%$ der befragten Unternehmen genutzt, was für diese relativ „,neuen“ Ansätze sehr hoch ist, auch wenn sie letztlich auf sehr traditionellen Konzepten wie der AIDA-Formel beruhen. Diese insbesondere in Unternehmensberatungsprozessen beliebten Funnelanalysen (Brand Purchase Funnel) (Riesenbeck und Perrey 2004, S. 100 ff.; Bauer et al. 2016, S. 40 ff.) sind ein Instrument des strategischen Marketingcontrollings (siehe ausführlich Reinecke 2021). Der Trichter visualisiert dabei konkurrenzorientiert Schwachstellen im Kundenkaufprozess: An welcher Stelle gehen im Benchmarking besonders viele (potenzielle) Käufer oder Kunden verloren? Der statische Markenfunnel wurde im Laufe der Jahre zu einer dynamischeren Kaufprozessanalyse (Decision Journey Analysis) weiterentwickelt (Edelman und Singer 2015).

Hingegen wird die klassische Szenariotechnik nur unregelmäßig eingesetzt. Ein Grund hierfür könnte die Subjektivität sowie der damit einhergehende wahrgenommene „esoterische Charakter“ von Zukunftsprognosen sein. Beim Thema Marktsegmentierung offenbart sich ein großer Gegensatz zwischen Wissenschaft und Praxis: Während die Notwendigkeit einer fundierten Marktsegmentierung in der Marketingliteratur weitgehend unstrittig ist, verzichten viele Unternehmen auf den regelmäßigen Einsatz von Segmentierungsstudien. Dies kann als ein Indiz dafür gewertet werden, dass Unternehmen nach wie vor Schwierigkeiten haben, eine Segmentierung von der Planung bis zur Implementierung in Kommunikation und Verkauf erfolgreich umzusetzen (Belz 2009, S. 79 f.). Marketingaudits finden ebenfalls in wenigen der befragten Unternehmen regelmäßige Anwendung. Eine solche umfassende, systematische Untersuchung von Marketingumwelt, -zielen, -strategien sowie von Marketingprozessen, -organisation und -maßnahmen einer strategischen Geschäftseinheit dient dazu, Herausforderungen und Chancen des Marketing aufzudecken und einen Maßnahmenplan für eine bessere Marketingleistung zu generieren (siehe ausführlich Reinecke und Janz 2007, S. 146 ff. und Reinecke 2014). Da Marketingaudits hauptsächlich situationsspezifisch, beispielsweise bei einem Führungswechsel im Gesamtunternehmens oder im Marketingbereich veranlasst werden, überrascht ihr unregelmäßiger Einsatz in den befragten Unternehmen nicht.

Neben der beschreibenden Analyse der Einsatzhäufigkeit der Controllinginstrumente wurde analysiert, ob sich erfolgreiche Unternehmen in dieser Hinsicht von 
weniger erfolgreichen unterscheiden. Grundlage für diese Analysen waren drei Erfolgsindizes. Der erste Erfolgsindex ergibt sich aus der Summe der jeweils wahrgenommen Zielerreichung bezüglich der vier Dimensionen der Balanced Scorecard (jeweils unternehmensspezifisch gewichtet zur angegebenen Bedeutung der Dimension). Den zweiten Erfolgsindex bildet die Summe der jeweils wahrgenommen Zielerreichung bezüglich der vier Kernaufgaben des aufgabenorientierten Ansatzes (jeweils unternehmensspezifisch gewichtet zur angegebenen Bedeutung der Kernaufgabe). Die Summe der jeweils wahrgenommenen Zielerreichung bezüglich Wachstum, Profitabilität und Risikominimierung (jeweils unternehmensspezifisch gewichtet zur angegebenen Zielbedeutung) bildet den dritten Erfolgsindex.

Anschließend wurde ein Quartilsvergleich durchgeführt, indem die jeweils $25 \%$ (subjektiv) erfolgreichsten Unternehmen mit den $25 \%$ wenigsten erfolgreichen Unternehmen hinsichtlich ihrer Einsatzintensität von strategischen Controllinginstrumenten verglichen wurden (siehe Abb. 13).

Die Ergebnisse zeigen, dass erfolgreiche Unternehmen stärker auf Marketingaudits, Mitarbeiterzufriedenheitsmessungen, Trichtermodelle, Image- und Positionierungsstudien sowie Kunden- und Produktportfolios setzen. Die hochsignifikant höhere Einsatzintensität von strategischen Kundenportfolios und Trichtermodellen bei Unternehmen, die hinsichtlich der Erfüllung der Marketingkernaufgaben besonders erfolgreich sind, deutet auf eine stärkere Kundenzentrierung dieser Unternehmen hin. Noch mal sei darauf hingewiesen, dass zwischen Instrumenteneinsatz und Erfolg kein einfacher Ursache-Wirkungszusammenhang bestehen muss: Es ist auch denkbar, dass die erfolgreichen Unternehmen die geschilderten Marketingcontrollinginstrumente nur deshalb überdurchschnittlich häufig einsetzen, weil sie dank ihres Erfolgs über höhere finanzielle Mittel für das Marketingcontrolling verfügen. Dennoch zeigt sich: Controllingintensität und Erfolg gehen miteinander einher. Dies

\begin{tabular}{|l|r|c|}
\hline \multicolumn{1}{|c|}{$\begin{array}{c}\text { Einsatz des strategischen } \\
\text { Controllinginstruments }\end{array}$} & $\begin{array}{c}\text { T-Test } \\
\text { Sig. }\end{array}$ & $\begin{array}{c}\text { Signifikanzniveau } \\
\text { *signifikant }(p<=0.05) \\
* * \text { hochsignifikant }(p<=0.01) \\
* * * \text { höchstsignifkant }(p<=0.001)\end{array}$ \\
\hline \multicolumn{2}{|c|}{ Erfolgsindex Balanced Scorecard } \\
\hline Marketingaudits & 0.022 & $*$ \\
\hline Mitarbeiterzufriedenheitsmessungen & 0.046 & $*$ \\
\hline \multicolumn{2}{|c|}{ Erfolgsindex Aufgabenorientierter Ansatz } \\
\hline Image und Positionierungsstudien & 0.023 & $*$ \\
\hline Strategische Kundenportfolios & 0.004 & $*$ \\
\hline Strategische Produktportfolios & 0.05 & $*$ \\
\hline Mitarbeiterzufriedenheitsmessungen & 0.021 & $*$ \\
\hline Trichtermodell & 0.01 & $*$ \\
\hline \multicolumn{2}{|c|}{ Erfolgsindex Wachstums-, Profitabilitäts- und Risikominimierungsiele } \\
\hline Trichtermodell & 0.019 & $*$ \\
\hline
\end{tabular}

Abb. 13 Von erfolgreichen Unternehmen signifikant häufiger eingesetzte strategische Controllinginstrumente. (Quelle: eigene Darstellung) 
ist ein wesentliches Indiz dafür, dass kunden- und mitarbeiterorientierte Ansätze des strategischen Marketingcontrollings stärker gewichtet werden sollten.

\subsubsection{Controlling der Marketinginstrumente}

Das operative Marketingcontrolling bezieht sich primär auf die vier klassischen Marketinginstrumente: Marktleistungs- und Preisgestaltung, Kommunikation und Distribution. Die Ergebnisse (siehe Abb. 14) zeigen, dass im operativen Marketingcontrolling das traditionell teuerste Marketinginstrument besonders im Fokus steht: der persönliche Verkauf. Im Vergleich zu früheren Untersuchungen offenbart sich zudem, dass das Online-Marketingcontrolling deutlich an Bedeutung gewonnen hat - dies dürfte insbesondere auf den gestiegenen Anteil von Online-Maßnahmen am Marketingbudget und auf den guten Datenzugang mittels Online-Tools zurückzuführen sein. Auch Customer Journey-Analysen werden von mehr als $60 \%$ der Unternehmen zumindest gelegentlich eingesetzt.

Auffällig ist die recht stark ausgeprägte Erfolgsanalyse von Messen und Events. Diese Instrumente stehen sehr häufig hinsichtlich ihrer Wirksamkeit in der Kritik, so dass ein verstärktes Controlling dieser Formen der „Live Communication“ nachvollziehbar ist - zumal viele Industrieunternehmen über $50 \%$ ihres Marketingbudgets für Messen und andere Live Communication-Maßnahmen ausgeben.

Die klassische Marketingkommunikation wird, mit Ausnahme der Social Media Kommunikation, lediglich vereinzelt intensiv gesteuert. Auf den ersten Blick erscheint dies aufgrund des nach wie vor durchaus hohen Anteils am Gesamtmarketingbudget verwunderlich. Allerdings sind die Kosten für ein professionelles Werbebzw. Kommunikationscontrolling relativ hoch, weshalb es weitgehend nur von größeren Unternehmen intensiv betrieben wird (siehe Abb. 15). Weitere Gründe für das niedrige Engagement im Werbe- und Kommunikationscontrolling dürften die dafür erforderliche hohe Methodenkompetenz sowie grundsätzliche Vorbehalte ge-

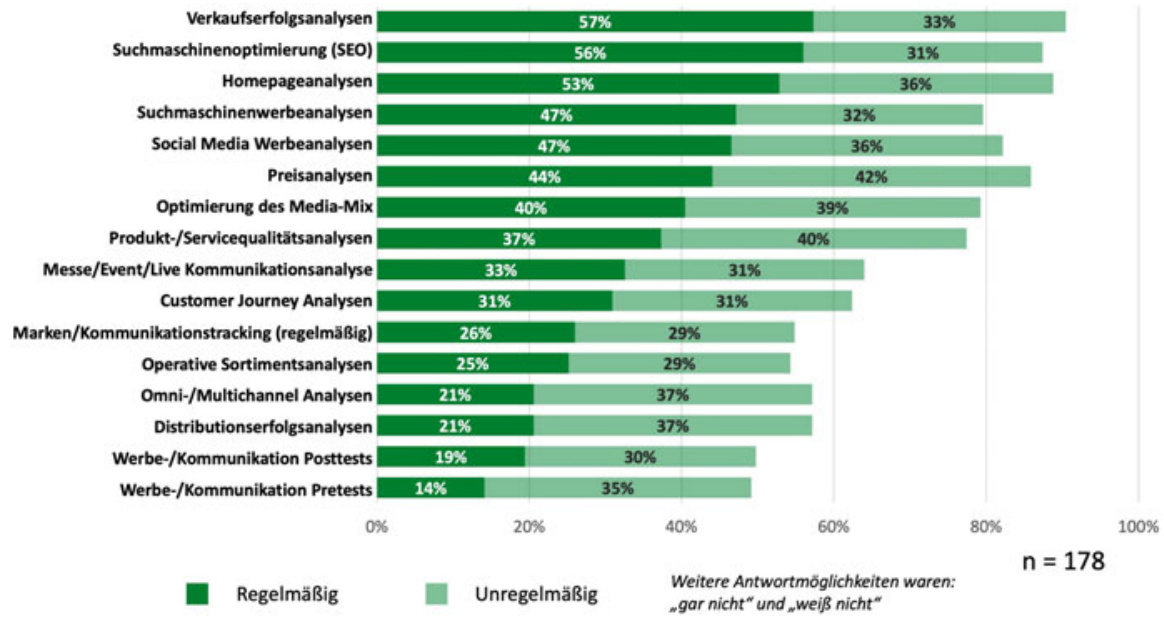

Abb. 14 Einsatz operativer Marketinginstrumente. (Quelle: eigene Darstellung) 


\begin{tabular}{|l|c|c|}
\hline $\begin{array}{c}\text { Einsatz des operativen Controlling } \\
\text { Instruments }\end{array}$ & Sig. & $\begin{array}{c}\text { Signifikanzniveau } \\
\text { *signifikant }(p<=0.05) \\
* * \text { hochsignifikant }(p<=0.01) \\
* * * \text { höchstsignifkant }(p<=0.001)\end{array}$ \\
\hline Produkt/Service Qualitätsanalysen & 0.003 & $* *$ \\
\hline Operative Sortimentsanalysen & 0.002 & $* *$ \\
\hline Preisanalysen & 0.000 & $* * *$ \\
\hline Werbe/Kommunikation Pretests & 0.001 & $* * *$ \\
\hline Werbe/Kommunikation Posttests & 0.000 & $* * *$ \\
\hline Markenkommunikationstracking & 0.000 & $* * *$ \\
\hline Suchmaschinewerbeanalysen & 0.000 & $* * *$ \\
\hline Customer Journey Analysen & 0.004 & $* *$ \\
\hline Distributionserfolgsanalysen & 0.004 & $* * *$ \\
\hline Omni/Multichannelanalysen & 0.000 & \\
\hline
\end{tabular}

Abb. 15 Quartilsvergleich der Einsatzintensität von operativen Controllinginstrumenten zwischen den größten und kleinsten Unternehmen (gemessen an der Mitarbeiteranzahl). (Quelle: eigene Darstellung)

genüber der Möglichkeit eines Kommunikationscontrollings sein. Werbe-Pretests werden beispielsweise lediglich von $14 \%$ der befragten Unternehmen regelmäßig eingesetzt, obwohl diese bei großen Kampagnen zumindest zur Überprüfung der Verständlichkeit der Botschaft sehr empfehlenswert sind. Aber auch Ad hoc-Posttests erfolgen nur bei $19 \%$ der Unternehmen regelmäßig. Bedenkenswert ist ferner, dass lediglich $18 \%$ ein regelmäßiges Kommunikationstracking durchführen - denn ein unregelmäßiges Tracking ist kaum zielführend und folglich nicht sinnvoll. Auch für ein professionelle Markenführung ist das Tracking ein wichtiges Fundament.

Somit zeigt sich, dass lediglich jene Instrumente des Marketingcontrollings stark zum Einsatz kommen, die ohnehin häufig aus Standardmarktforschungsstudien, aus Online-Tools, aus dem Qualitätsmanagement oder aus finanzwirtschaftlichen Routinereports vorliegen. Eine weitere Baustelle bleibt die Durchführung von Omni-/ Multi-Channel-Analysen; gerade in Zeiten integrierter Marketing- und Distributionsansätze offenbart sich hier Handlungsbedarf.

Im Rahmen tiefer gehender Analysen wurde untersucht, in wie weit sich Großvon Kleinunternehmen hinsichtlich der Einsatzintensität von operativen Controllinginstrumenten unterscheiden. Mittels eines Quartilsvergleichs wurden die $25 \%$ (gemessen an der Mitarbeiterzahl) größten befragten Unternehmen mit den $25 \%$ kleinsten Unternehmen verglichen. Die Ergebnisse zeigen deutlich, dass das operative Marketingcontrolling bei allen vier klassischen Marketinginstrumenten (Marktleistungs- und Preisgestaltung, Kommunikation und Distribution) von Großunternehmen signifikant regelmäßiger betrieben wird (siehe Abb. 15). Dies dürfte zum einen auf die geringeren verfügbaren finanziellen Mittel von Kleinunternehmen zurückzuführen sein. Zum anderen lassen die Ergebnisse aber auch vermuten, dass es an einfachen, pragmatischen und kosteneffizienten operativen Controllingverfah- 


\begin{tabular}{|c|c|c|}
\hline \multicolumn{3}{|c|}{ Erfolgsindex Aufgabenorientierter Ansatz } \\
\hline $\begin{array}{l}\text { Einsatz des operativen } \\
\text { Controllinginstruments }\end{array}$ & $\begin{array}{l}\text { T-Test } \\
\text { Sig. }\end{array}$ & $\begin{array}{c}\text { Signifikanzniveau } \\
\text { *signifikant }(p<=0.05) \\
{ }^{* *} \text { hochsignifikant }(p<=0.01) \\
* * * \text { höchstsignifkant }(p<=0.001)\end{array}$ \\
\hline Produkt/Service Qualitätsanalysen & 0.004 & ** \\
\hline Preisanalysen & 0.020 & $*$ \\
\hline Werbe/Kommunikation Posttests & 0.015 & $*$ \\
\hline Markenkommunikationstracking & 0.005 & ** \\
\hline SEO & 0.005 & $* *$ \\
\hline Social Media Werbeanalysen & 0.002 & $* *$ \\
\hline Homepageanalysen & 0.001 & $* * *$ \\
\hline Customer Journey Analysen & 0.011 & $*$ \\
\hline Optimierung des Media Mix & 0.000 & $* * *$ \\
\hline Distributionserfolgsanalysen & 0.021 & * \\
\hline Omni/Multichannelanalysen & 0.000 & *** \\
\hline
\end{tabular}

Abb. 16 Von erfolgreichen Unternehmen (Erfüllung der Kernaufgaben des aufgabenorientierten Ansatzes) überdurchschnittlich häufig eingesetzte operative Instrumente. (Quelle: eigene Darstellung)

ren fehlt, die von mittelständischen- und Kleinunternehmen regelmäßig eingesetzt werden können.

Zudem wurde untersucht, ob sich die erfolgreichsten $25 \%$ der Unternehmen von den $25 \%$ wenigsten erfolgreichen hinsichtlich des operativen Instrumenteneinsatzes unterscheiden. Zur Quartilsbildung wurde erneut der Erfolgsindex des aufgabenorientierten Ansatzes (Zielerreichung bzgl. Kundenakquisition und -bindung sowie Leistungsinnovation und -pflege) als Erfolgsindikator herangezogen, weil dieser spezifischer auf das operative Marketing eingeht als gesamtunternehmerische Zielsysteme. Abb. 16 zeigt die entsprechenden Ergebnisse. Dabei offenbart sich eindrucksvoll, dass Unternehmen, die hinsichtlich der Erfüllung der vier Marketingkernaufgaben besonders erfolgreich sind, sich insbesondere bei der Controllingintensität von Omni- und Multichannelanalysen sowie der Media Mix-Optimierung von weniger erfolgreichen Unternehmen unterscheiden. Die Ergebnisse unterstreichen die Notwendigkeit, neben medienspezifischen Aspekten auch die Wirkungen des gesamten Marketing-Mix zu analysieren. Nur eine integrierte Steuerung der Marketingmaßnahmen erzeugt instrumentenübergreifende Synergien und ermöglicht eine effektive Umsetzung der Marketingstrategie. Erfolgreiche Unternehmen setzen zudem verstärkt auf Homepageanalysen und auf ein intensiveres Kommunikationscontrolling. Auch wenn hier ebenfalls kein einfacher Ursache-Wirkungszusammenhang zwischen dem Einsatz operativer Instrumente und dem Erfolg unterstellt werden kann, so ist dennoch zumindest zu vermuten, dass die Professionalität des operativen Controllings einen gewissen positiven Einfluss auf das Erreichen der Marketingziele hat. 


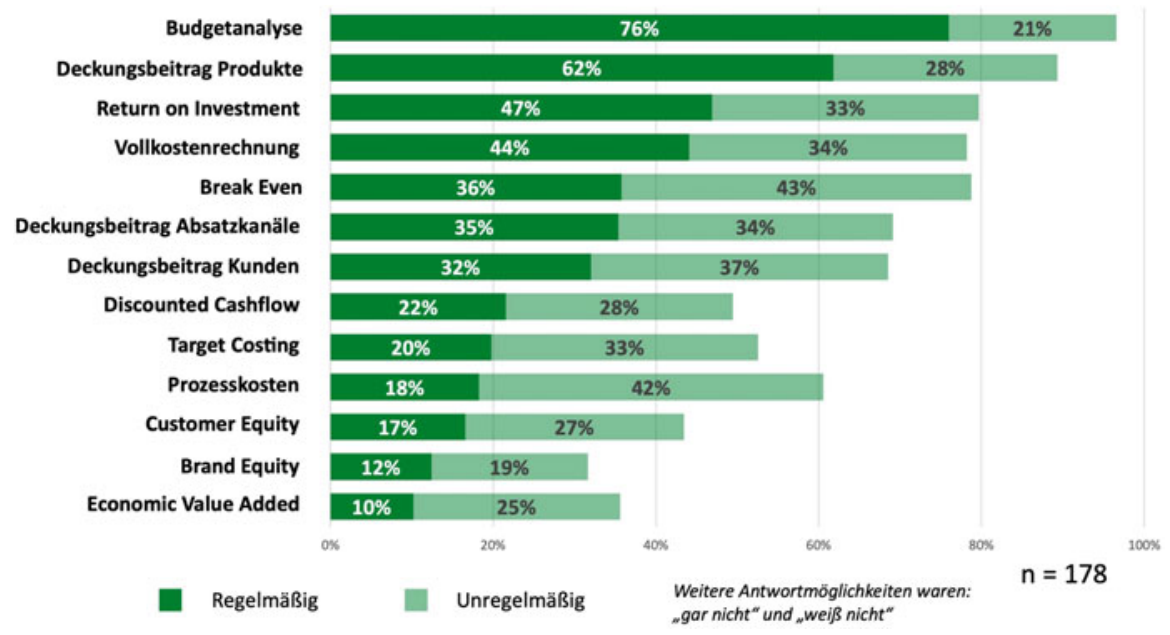

Abb. 17 Einsatzintensität von Instrumenten des Marketingaccountings. (Quelle: eigene Darstellung)

\subsubsection{Marketingaccounting}

Instrumente des Kosten- und Erfolgscontrollings haben einen direkten finanzwirtschaftlichen Bezug. Wie Abb. 17 zeigt, ist die Budgetanalyse mit $76 \%$ der absolute Spitzenreiter bei den Instrumenten des Marketingaccountings. Ohne die Bedeutung dieses wichtigen Controllinginstruments schmälern zu wollen, stellt sich angesichts der bereits diskutierten relativ geringen Professionalität bei der Bestimmung der Höhe des Marketingbudgets (siehe Abb. 7) die Frage, ob diese einseitige Schwerpunktsetzung zielführend ist. Zwar ist dieses Verhalten nachvollziehbar, weil das Marketingbudget häufig zu den wenigen finanzwirtschaftlichen Zahlen zählt, die das Finanzcontrolling aus dem Bereich Marketing erhält - und letzteres somit verständlicherweise einen Fokus auf die Kontrolle dieser Kostenaspekte legt. Wenn allerdings - wie dargelegt - die Marketingbudgetierung primär erfahrungs- und vergangenheitsorientiert erfolgt, erscheint eine prioritäre Ausrichtung des Controllings auf Budgetanalysen als eher fragwürdig, weil dann Fragen der Effizienz (,Werden die Budgets eingehalten?“) gestellt werden, bevor die Fragen der Effektivität („Wie hoch sollte das Marketingbudget sein? In welche Instrumente und Bereiche sollte es investiert werden?") beantwortet wurden. Mit anderen Worten: Intensive Budgetanalysen sind wichtig, aber nur dann wirklich sinnvoll, wenn auch die Marketingbudgetierung professionell erfolgt.

Neben der Budgetanalyse gehören Deckungsbeitragsrechnungen in Bezug auf Produkte/Aufträge/Projekte (62\%) sowie Investitionsrechnungen (47\%) und Vollkostenrechnungen (44 \%) aus der Sicht der befragten Führungskräfte zu den meistbenutzten Instrumenten. Diese Instrumente des Marketingaccountings standen vor dreißig Jahren im Zentrum des Marketingcontrollings, weil sie damals - vor der Einführung betriebswirtschaftlicher Standardsoftware - einen zentralen Informationsengpass vieler Unternehmen betrafen; heutzutage liefern die informationstech- 
nischen Controllinglösungen weitgehend zufriedenstellende Standardauswertungen. Allerdings offenbart sich noch eine gewisse Lücke als Herausforderung: Die Marketingplanung ist in den letzten Jahrzehnten deutlich kunden(gruppen)orientierter geworden (Homburg et al. 2008); dennoch werden kundenorientierte Deckungsbeitragsrechnungen lediglich bei etwas weniger als einem Drittel der Unternehmen regelmäßig eingesetzt, was auf erhebliche Defizite im operativen Marketingaccounting schließen lässt. Wie lässt sich kundenorientiertes Marketing planen und umsetzen, wenn das Controlling keine Informationen darüber liefert, welche die tatsächlich rentablen Kunden(gruppen) eines Unternehmens sind? Dieser Mangel ist insbesondere aufgrund des großen Anteils an Dienstleistungsunternehmen in der Stichprobe schwerwiegend: Gerade für Dienstleister ist ein kundenorientiertes Marketingcontrolling besonders wichtig, da die Profitabilität der Leistung maßgeblich durch das Mitwirken des Kunden beeinflusst wird. Auch Markenwertberechnungen werden lediglich in $12 \%$ der befragten Unternehmen regelmäßig eingesetzt. Dies kann einerseits darauf zurückzuführen sein, dass es nach wie vor keinen verlässlichen Methodenstandard gibt, der den (Praktikabilitäts-)Anforderungen der Praxis gerecht wird. Zum anderen ist ein auf den Markenwert fokussiertes Controlling bei kleineren Unternehmen häufig aus wirtschaftlichen Gründen nicht angemessen.

Insgesamt dominieren im Marketingaccounting nach wie vor traditionelle, „bewährte" Methoden. Neuere und methodisch anspruchsvollere Verfahren werden scheinbar als riskant, zu aufwändig oder zu teuer erachtet; oder aber es fehlt in der Unternehmenspraxis noch das entsprechende Know-how. Dennoch sind es gerade diese innovativen Marketingcontrollinginstrumente, welche eine Quantifizierung und Monetarisierung von Marketingmaßnahmen ermöglichen und somit Unternehmen dem Ziel einer wertorientierten Marketingführung näherbringen würden.

\section{$5 \quad$ Fazit: Ohne Marketingplanung kein Marketingcontrolling}

Die empirischen Ergebnisse belegen, dass einerseits ein großes Bedürfnis danach besteht, den „Return on Marketing“ und somit die Effektivität und Effizienz im Marketing sicherzustellen und nachzuweisen. Andererseits mangelt es nach wie vor an der Implementierung geeigneter Instrumente, die dabei helfen, sowohl den Marketingführungsprozess als auch die Wirtschaftlichkeit von Marketingmaßnahmen zu optimieren. Die Marketingrealität ist von einem echten wert- bzw. investitionsorientierten Marketing somit noch weit entfernt.

Forderungen nach einem verbesserten Reporting an das Top Management ergeben allerdings wenig Sinn, wenn diese nicht durch den Aufbau von Fach- und Methodenkompetenz begleitet werden. Planung ohne Controlling ist nur beschränkt sinnvoll. Folglich erscheint es ratsam, einen Mindestanteil des Marketingbudgets für eine Optimierung des Marketingcontrollings einzusetzen. Eine gewisse Investition knapper Marketingmittel zur Evaluation von Möglichkeiten zur Steigerung der Marketingeffektivität und -effizienz ist speziell dort sinnvoll, wo bisher weitgehend auf Feedback im Sinne eines Lernprozesses verzichtet wurde. Handlungsbedarf besteht insbesondere bei der Professionalisierung der Marketingbudgetierung, dem Kommunikationscon- 
trolling, dem Einsatz differenzierter Marketingkenngrößen sowie einem kunden(gruppen) orientierten Marketingaccounting. Im Bereich des digitalen Marketing ist die Datenverfügbarkeit deutlich besser im Vergleich zum traditionellen Marketing, was zu einem überdurchschnittlichen Einsatz des Marketingcontrollings in diesem Bereich führt.

Andererseits lässt sich aus den Ergebnissen auch eine Kritik an der betriebswirtschaftlichen Forschung herauslesen: Controlling- und Marketingwissenschaft sind herausgefordert, Verfahren zu entwickeln, die auch von mittelständischen Unternehmen eingesetzt werden können, um ihre Marketing Performance zu erhöhen. Die größte Herausforderung bleibt nach wie vor die Ursache-Wirkungsbeziehung einzelner Marketingmaßnahmen im Feld, das heißt der unternehmerischen Realität, zu ermitteln.

\section{Zusammenfassung: Marketingcontrolling 2021}

- Die Ergebnisse der vorliegenden Studie basieren auf einer schriftlichen OnlineBefragung im deutschsprachigen Raum (Schwerpunkt Schweiz), an der 179 Führungskräfte teilgenommen haben. Die branchenübergreifende Studie fand im Sommer 2020 statt; sie erhebt keinen Anspruch auf Repräsentativität.

- Die Notwendigkeit, den „Return on Marketing“ nachzuweisen, ist in den letzten drei Jahren deutlich gestiegen. Marketingführungskräfte planen verstärkt Daten, Analysen und Fakten zur Unterstützung von Marketingentscheidungen einzusetzen.

- Die Funktion des Marketingcontrollings besteht darin, die Effektivität (Wirksamkeit) und Effizienz (Wirtschaftlichkeit) einer marktorientierten Unternehmensführung sicherzustellen. Die Zufriedenheit mit dem Marketingcontrolling ist insgesamt bescheiden und bei wenigen Unternehmen besonders hoch.

- Die größten Herausforderungen des Marketingcontrollings sind der Nachweis von Ursache-Wirkungsbeziehungen einzelner Marketingmaßnahmen, die Messbarkeit des Marketingerfolgs, fehlendes Know-how und Ressourcen bezüglich Datenanalyse \& -auswertung, Datenzugang \& -erhebung sowie Informationstechnologie. Ferner werden für das Marketingcontrolling häufig unzureichend finanzielle Mittel bereitgestellt.

- Eine begründete, rationalitätsgesteuerte Marketingbudgetierung fehlt bei den meisten Unternehmen.

- Finanzwirtschaftliche Ziele werden in der Unternehmenspraxis priorisiert. Dabei werden Wachstums- und Profitabilitätsziele von Marketingführungskräften ungefähr gleich gewichtet.

- Einfache, „klassische“ Marketing- und Verkaufskennzahlen (zumeist absatz- und finanzwirtschaftliche Größen wie Umsatz, Absatz, Nettogewinn und Umsatzrentabilität) kommen in der Unternehmenspraxis häufig zum Einsatz. Kundenorientierte Kennzahlen werden ebenfalls erhoben, allerdings seltener als Top-Steuerungskennzahlen. Konkurrenzorientierte Kenngrößen werden (mit Ausnahme des Marktanteils) dagegen kaum erhoben.

- Betriebswirtschaftlicher Erfolg und der Einsatz ausgewählter finanzieller und nicht-finanzieller Marketingkennzahlen gehen miteinander einher, ohne dass 
jedoch eine einfache Ursache-Wirkungskette bestehen muss. Unternehmen, die komplexere Kennzahlen wie den Kundenbindungsindex oder den finanziellen Kundenwert erheben, zeigen eine signifikant höhere Zufriedenheit mit dem unternehmensinternen Marketingcontrolling.

- Beim strategischen Marketingcontrolling zeigt sich, dass besonders erfolgreiche Unternehmen Customer Scoring-Modelle, Marketingaudits, Mitarbeiterzufriedenheitsmessungen und Trichtermodelle intensiver einsetzen als weniger erfolgreiche Unternehmen.

- Beim operativen Marketingcontrolling kommen überwiegend jene Instrumente stark zum Einsatz, die ohnehin häufig aus Standardmarktforschungsstudien, aus Online-Tools, aus dem Qualitätsmanagement oder aus finanzwirtschaftlichen Routinereports vorliegen. Eine große Baustelle ist das unterentwickelte Controlling der klassischen Marketingkommunikation. Erfolgreiche Unternehmen unterscheiden sich von weniger erfolgreichen insbesondere bei der Häufigkeit der Durchführung von Omni/Multichannelanalysen und der Optimierung des Media Mix.

- Im Marketingaccounting gehören neben der Budgetanalyse Deckungsbeitragsrechnungen bezüglich Produkten/Aufträgen/Projekten, Investitionsrechnungen und Vollkostenrechnungen zu den Standardinstrumenten. Speziell für Dienstleister wäre jedoch ein kundenorientiertes Marketingaccounting besonders wichtig, insbesondere Kundendeckungsbeitragsrechnungen.

- Marketingplanung ohne Marketingcontrolling ist sinnlos, Marketingcontrolling ohne eine differenzierte Marketingplanung aber gar nicht erst möglich. Ziel des Marketingcontrollings ist es immer, Lernprozesse im Marketing zu bewirken, um die Marketing Performance zu erhöhen.

\section{Literatur}

Bauer, T., Freundt, T., Gordon, J., Perrey, J., \& Spillecke, D. (2016). Marketing performance. How marketers drive profitable growth. Chichester (UK): Wiley.

Belz, C. (2009). Marketing gegen den Strom. St. Gallen: Thexis.

Bendle, N. T., Farris, P. W., Pfeifer, P. E., \& Reibstein, D. J. (2020). Marketing metrics. The manager's guide to measuring marketing performance (4. Aufl.). Upper Saddle: Pearson.

Bonoma, T. V., \& Clark, B. H. (1988). Marketing performance assessment. Boston: Harvard Business School Press.

Deyhle, A. (1988). Marketingcontrolling - Das Denken vom Kunden her. Controller Magazin, 12(1), 15-20.

Edelman, D., \& Singer, M. (2015). Competing on customer journeys. Harvard Business Review, 11, 4-11.

Fuchs, D. (2008). Marketingbudgetierung: konzeptionelle Grundlagen und empirische Befunde. St. Gallen/Bamberg: Difo-Druck.

Gigerenzer, G., \& Brighton, H. (2009). Homo heuristicus: Why biased minds make better inferences. Topics in Cognitive Science, 1, 107-143.

Hanssens, D., \& Pauwels, K. (2016). Demonstrating the value of marketing. Journal of Marketing, $80,172-190$.

Homburg, C., Droll, M., \& Tozek, D. (2008). Customer prioritization: Does it pay off, and how should it be implemented? Journal of Marketing, 72(5), 110-130. 
Horváth, P. (1985). Die Aufgaben des Marketing-Controllers. In R. S. Eschenbach (Hrsg.), Marketing controlling, Österreichischer Controllertag (S. 7-29). Wien: Service, Fachverlag an der Wirtschaftsuniversität Wien.

Kaplan, R. S., \& Norton, D. P. (1992). The balanced scorecard - measures that drive performance. Harvard Business Review, 70(1), 71-79.

Katsikeas, C. S., Morgan, N. A., Leonidou, L. C., \& Hult, G. T. M. (2016). Assessing performance outcomes in marketing. Journal of Marketing, 80, 1-20.

Lasslop, I. (2003). Effektivität und Effizienz von Marketing-Events. Wiesbaden: Gabler.

Reinecke, S. (2004). Marketing Performance Management. Empirisches Fundament und Konzeption für ein integriertes Marketingkennzahlensystem. Wiesbaden: Gabler.

Reinecke, S. (2010). Marketingcontrolling, Sicherstellen von Effektivität und Effizienz einer marketingorientierten Unternehmensführung. Controlling, 22(2), 75-82.

Reinecke, S. (2014). Return on marketing 2014. Sicherstellen der Marketing Performance in der Praxis - Empirische Ergebnisse und Implementierungshinweise zum Marketing- und Verkaufscontrolling. St. Gallen: Institut für Marketing.

Reinecke, S. (2020). Marketingentscheidungen: Der Einfluss künstlicher Intelligenz auf den Management-Entscheidungsprozess. In M. Bruhn, M. Kirchgeorg \& C. Burmann (Hrsg.), Marketing Weiterdenken - Zukunftspfade für eine marktorientierte Unternehmensführung (2. Aufl., S. 437-452). Wiesbaden: Springer Gabler.

Reinecke, S. (2021). Marketing-Controlling: Marketingeffektivität und -effizienz sicherstellen. In R. Eschenbach, J. Baumüller \& H. Siller (Hrsg.), Funktions-Controlling - Praxishandbuch für Unternehmen, Non-Profit-Organisation und die öffentliche Verwaltung. Wiesbaden: Springer. im Druck.

Reinecke, S., \& Eberharter, J. (2010). Marketingcontrolling 2010. Einsatz von Methoden und Verfahren des Marketingcontrollings in der Praxis. Controlling - Zeitschrift für erfolgsorientierte Unternehmenssteuerung, 22(8/9), 438-447.

Reinecke, S., \& Geis, G. (2020). Kennzahlensysteme in Marketing \& Verkauf: Gütekriterien, Grundprinzipien, Implementierung. In C. Zerres (Hrsg.), Handbuch Marketing-Controlling (5. Aufl.). Wiesbaden: Springer Gabler. (im Druck).

Reinecke, S., \& Janz, S. (2007). Marketingcontrolling. Sicherstellen von Effektivität und Effizienz des Marketing. Stuttgart: Kohlhammer.

Riesenbeck, H., \& Perrey, J. (2004). Mega-Macht Marke. Frankfurt a. M.: Ueberreuter Wirtschaftsverlag.

Sheth, J. N., \& Sisodia, R. S. (1995). Feeling the heat. Marketing Management, 4(2), 9-23.

Sheth, J. N., \& Sisodia, R. S. (2002). Marketing productivity - issues and analysis. Journal of Business Research, 55, 349-362.

Siegwart, H., Reinecke, S., \& Sander, S. (2010). Kennzahlen für die Unternehmensführung (7. Aufl.). Bern: Haupt.

Stewart, D. W., \& Gugel, C.T. (2016). Accountable marketing. Linking marketing actions to financial performance (1. Aufl.). Oxfordshire: Routledge.

Tomczak, T., \& Reinecke, S. (1996). Der aufgabenorientierte Ansatz. Eine neue Perspektive für das Marketing-Management. Fachbericht für Marketing. St. Gallen.

Tomczak, T., Kuß, A., \& Reinecke, S. (2014). Marketingplanung, Einführung in die marktorientierte Unternehmens- und Geschäftsfeldplanung (7. Aufl.). Wiesbaden: Springer Gabler. 